National Aeronautics and Space Administration

Contract No. NASw-6

Technical Release No. 34-12

EXPLORER SATELLITE ELECTRONICS

Walter K. Victor

Henry L. Richter

John P. Eyraud

72

Copy No.

This document consists of cover and 35 leaves.

\author{
JET PROPULSION LABORATORY \\ A Research Facility of \\ National Aeronautics and Space Administration \\ Operated by \\ California Institute of Technology \\ Pasadena, California \\ January 29, 1960
}




\section{CONTENTS}

Page

I. Introduction ....................... 1

III. Design Restrictions Imposed by the Vehicle and Orbit . . . . . 1

III. Explorer IGY Instrumentation . . . . . . . . . . . . . 3

IV. Cosmic-Ray Instrumentation and Measurements . . . . . . . 5

V. Micrometeorite Instrumentation and Measurements . . . . . . 9

VI. Telemetering Subcarrier Oscillators . . . . . . . . . 11

VII. Microlock Communications System . . . . . . . . . . . 12

VIII. Microlock Ground Receiver . . . . . . . . . . . . . 13

IX. Satellite Transmitters . . . . . . . . . . . . . 15

X. Satellite Antennas ................... . 16

XI. Preflight Testing. . . . . . . . . . . . . . . 18

\section{FIGURES}

1. The United States' First Earth Satellite, Explorer I . . . . . 20

2. Instrumentation Installed in Explorer I . . . . . . . . . . 21

3. Instrumentation Installed in Explorer III . . . . . . . . . . 22

4. Tape Recorder for Cosmic-Ray Data Flown in Explorer III . • 23

5. Telemetering Systems Flown in Explorer I. . . . . . . . . 24

6. Cosmic-Ray Data: (a) Pulse Inputs to Recorder; (b) Typical

Reduced Counting Rates for One Complete Orbit . . . . . . . 25

7. Wire-Gauge Micrometeorite Detector. . . . . . . . . . 26

8. Micrometeorite Microphone and Amplifier . . . . . . . . 26 
FIGURES (Cont'd)

Page

9. Subcarrier Oscillators Used in Explorer Satellites . . . . . . 27

10. Microlock Communications System Used in Tracking

Explorer Satellites . . . . . . . . . . . . . . . 28

11. Typical Recordings of Explorer Telemetry . . . . . . . . . . 29

12. Microlock Phase-Locked Ground Receiver and Interferometer ................... 30

13. Transmitters Used in Explorer Satellites: (a) Low-Power

Transmitter; (b) High-Power Transmitter . . . . . . . . . . 31 


\title{
EXPLORER SATELLITE ELECTRONICS ${ }^{*}$
}

\author{
Walter K. Victor \\ Henry L. Richter \\ John P. Eyraud
}

\begin{abstract}
A discussion is presented of the design restrictions and the philosophy which enabled the Explorer satellites to be first during the IGY to reveal the presence of a belt of intense cosmic radiation encircling the earth's equator. In addition, an indication of the amount and momentum of cosmic dust in the solar system was obtained from the Explorers. Methods used to obtain reliability in the transducing and communications system are described, together with interpretations of space-environment information as deduced from the narrow-band telemetry.
\end{abstract}

* Portions of the following report were originated under studies conducted for the Department of Army Ordnance Corps under Contract No. DA-04-495-Ord 18. Such studies are now conducted for the National Aeronautics and Space Administration under Contract No. NASw-6. 


\section{INTRODUCTION}

During the International Geophysical Year, the United States Army in cooperation with the Jet Propulsion Laboratory placed three Explorer satellites in orbit around the earth. Telemetered data from Explorers I, III, and IV provided new, unexpected knowledge and an increased understanding of the hazardous space environment encountered above the earth's atmosphere. National Academy of Sciences IGY instruments were adapted to the payload structure of Explorers I (Fig. 1) and III by engineers and technicians at JPL; Explorer IV was assembled at the Army Ballistic Missile Agency. Most of the scalers, telemetering oscillators, and transmitters had been designed and assembled at JPL. A world-wide satellite tracking network was based on a sensitive Microlock communications system originally developed by JPL for long-range tracking of tiny radio beacons inside re-entry nose cones.

\section{DESIGN RESTRICTIONS IMPOSED BY THE VEHICLE AND ORBIT}

In 1957, the most reliable American rocket vehicle capable of boosting a satellite up to a $200-\mathrm{mi}$ orbit altitude was the Army's Redstone guided missile. For the upper high-speed stages, another thoroughly tested Army rocket was available, a scaled-down version of the JPL-developed solid-propellant Sergeant missile. Both of these rockets had, however, been built for rugged tactical field handling and were structurally much heavier than if they had been designed specifically for satellite launching. On the other hand, the structure was proven trustworthy. Because of the structural weight, the maximum 
possible ratio of orbitable weight to launching weight was only about 1 to 1000 . This ratio meant that the final-stage rocket case and instrumented payload together could weigh no more than $31 \mathrm{lb}$. Of this weight, the rocket motor case used $12.7 \mathrm{lb}$ and the cylindrical instrument housing used $7.5 \mathrm{lb}$. Only $10.7 \mathrm{lb}$ could be allotted for all the instruments, the transmitters, the batteries, and their supporting structures. Severe restrictions were thus placed on individual component weights, battery power drain, and information bandwidth.

Internal temperatures of Explorer payloads had to be maintained within a range of -5 to $60^{\circ} \mathrm{C}$ in order to avoid freezing the mercury batteries or overheating the germanium transistors. Earth satellites are, however, subject to abrupt and severe temperature excursions about once every hour as they pass from direct sunlight into the earth's shadow and back into sunlight again. To slow down the heat-transfer rate between the steel shell and the internal payload electronics, Micarta was used for all supporting structures. Furthermore, to produce as long a thermal time constant as possible for all components, all electronics, including batteries, were thermally connected with one another. For midnight launchings, it was calculated that about $25 \%$ of the stainless steel outer surface should be coated with a heat-reflecting material to keep the average internal temperature at an optimum low operating level. Therefore, the nose and cylindrical shell of the instrument container were flame-sprayed with eight equally spaced aluminum oxide stripes.

Temperature data telemetered from the Explorers showed that the design objective had been met. Although the external shell temperatures fluctuated 
between -25 and $75^{\circ} \mathrm{C}$, the instruments remained within the safe 0 to $35^{\circ} \mathrm{C}$ range. The temperature of the satellite nose cone never rose above $40^{\circ} \mathrm{C}$.

\section{EXPLORER IGY INSTRUMENTATION}

The choice of instruments was critical in determining the success of the Explorer program. The instruments had to be scientifically valuable, capable of withstanding the comparatively rigorous vibration and spin environment of the Explorer rocket, and ready for immediate incorporation in November, 1957. The final selection was made by the IGY with the agreement of the experimenters, JPL and the Army Ordnance Missile Command.

A careful arrangement and mounting of these instruments was required for thermal and mechanical symmetry reasons. Arrangement of the instrumentation in Explorer I is shown in Fig. 2. The instrumentation included: (1) a Geiger-Mueller tube with its scaling circuit, which had been designed by Dr. James A. Van Allen of the State University of Iowa to measure the intensity of high-energy cosmic radiation; (2) four temperature-measuring devices, three on the outer shell of the satellite and one inside; (3) a sensitive microphone placed in contact with the shell, designed to respond to the impacts of micrometeorites; and (4) twelve external wire-grid erosion detectors, which had been designed by Dr. M. Dubin of the Air Force Cambridge Research Center to measure by their fracture the impact of micrometeorites. Since the maximum counting rate anticipated for cosmic rays was about 17 counts/sec, it was believed that a maximum reading rate of 80 counts/sec would be adequate. As it turned out, this rate was exceeded during each orbit. 
Explorer III, shown in Fig. 3, was designed to make more sophisticated measurements of cosmic-ray intensity. It carried the same type of GeigerMueller tube as Explorer I, but the information was stored on a magnetic tape. The tiny tape recorder (Fig. 4), whose maximum recording rate was 128 counts/sec, was designed by George Ludwig of SUI. Once during each orbit around the earth, when the satellite passed over one of the Minitrack receiving stations, a coded interrogation signal was transmitted to a special Minitrack receiver in the satellite. Upon receipt of this signal, a playback of the tape recorder was initiated. In approximately $5 \mathrm{sec}$, all of the cosmic-ray information gathered from one orbit around the earth was transmitted back to the Minitrack ground station. The transmitter used in the satellite for this operation was similar to the high-power transmitter of Explorer I.

Explorer IV, although still tiny by Russian standards, was nevertheless a fairly complex space laboratory. It carried a thin cesium iodide scintillation counter with a very thin window for detecting low-energy particles, a plastic scintillator for somewhat higher energy particles, a Geiger counter for still higher energy particles, and a lead-shielded Geiger counter for very high energy particles. The Geiger counters were reduced in size by a factor of 25 , and more scaling stages were included by $\mathrm{Dr}$. Van Allen to avoid the saturation problems which plagued the earlier two Explorers.

Block diagrams of the telemetering systems flown in the Explorer satellites are shown in Fig. 5. 


\section{COSMIC-RAY INSTRUMENTATION AND MEASUREMENTS}

The measurements of high-intensity radiation carried out in Explorers I, III, and IV were under the direction of Dr. Van Allen and his colleagues at the State University of Iowa who were responsible for the experiment design and for the scientific conclusions abstracted here. The intensity of cosmic radiation was measured by a standard Geiger-Mueller tube. An electronic scaling circuit was attached to the tube. In Explorer I, the output of a scale of 32 was telemetered directly. The maximum scaler counting rate was 4000 counts/sec; but the reduced telemetered data was readable only between $0.14 \mathrm{pulse} / \mathrm{sec}$ (one change of state per $2-\min$ pass) and $80 \mathrm{pulses} / \mathrm{sec}$.

In Explorer III, the tape-recorded information was handled as follows. A tuning fork and scaling circuit put out one pulse every second. This output is diagrammed in the first line at the top of Fig. 6a. The scaling circuit associated with the Geiger-Mueller tube put out one pulse for every 128 counts of the tube, as indicated for a possible case by the second line in Fig. 6a. Since the tape was advanced only once a second, the maximum rate that could be recorded was 128 counts/sec. Each pulse from the tuning fork was recorded on the tape unless it happened to follow an output from the Geiger-Mueller tube scaling circuit. In the latter case, the pulse from the cosmic-ray counting circuit blocked the next succeeding timer pulse. The result of this mixture was a series of pulses with some missing, as shown by the third line in Fig. 6a. The frequency of cosmic rays is then 128 divided by the number of timing pulses from blank to blank. 
Because of dead-time effects, when the counting rate is above 20,000 counts/sec, the $G-M$ tube responses become too weak to trigger the scaling circuit.

From a scientific viewpoint, the most startling information which has been generated by the Explorer satellites is the new cosmic-ray data. If a cosmic-ray Geiger counter is taken upward by rocket or balloon from the earth's surface to a point out in space, the intensity measured by the counter increases to a maximum of about 20 counts/sec at an altitude of $6 \mathrm{mi}$, then decreases to about 1 count/sec at an altitude of about $50 \mathrm{mi}$. Above this altitude it was expected to increase slowly to an asymptotic value of about 2 counts/sec at about $600 \mathrm{mi}$. When numerous tape recordings of Explorer I telemetry showed a sharp rise in cosmic-ray counting rate to the unbelievable 80 -count/sec rate, followed by an abrupt drop to zero for 5 to $15 \mathrm{~min}$ while the satellite was near apogee, it was at first presumed that the scaler was not functioning properly.

When the first cosmic-ray recordings from Explorer III started coming in, it seemed again that the scaler was not functioning properly. Reduced data from a typical Explorer III recording are shown in Fig. 6b. For the first $15 \mathrm{~min}$ the counting rate coincides closely with that expected. Suddenly there is a jump to the maximum possible recorder counting rate, 128 counts/sec. This is followed by an abrupt decrease to a zero counting rate. After about $15 \mathrm{~min}$ while no counts at all were being recorded, the rate again jumps to the maximum possible value. This rate continues for the next half hour, and then the count again suddenly falls to its expected level. This peculiar behavior was reproduced on almost every orbit, and each time over some portion of the orbit the counting rate appeared to be close to that expected. 
An analysis of several orbits shows that the sudden increase in counting rate and subsequent blocking of the counter occurred as the satellite approached its apogee and passed up through an altitude limit of approximately $600 \mathrm{mi}$. The counting rate returned to that expected as the satellite came back down through this limit on its way toward perigee. By testing a spare payload, it was found that saturation occurred at a counting rate of 20,000 counts/sec, which was 1000 times that expected. This intense radiation could not be electromagnetic. The 0.023 -in. steel shell of the satellite and the 0.05 -in. steel walls of the counting tube form a shield with a density of $1.5 \mathrm{~g} / \mathrm{cm}^{2}$. Photons with energies high enough to penetrate this quantity of material should have been noticed at much lower altitudes. Certainly they would have been recorded long before this by balloon flights and high-altitude rocket observations; but no such observations had been reported. On the other hand, high-altitude rocket measurements in the auroral zone had detected a similar type of radiation. Measurements on a spare payload indicated that the penetrating radiation could be produced by electrically charged particles such as electrons with energies above $3 \mathrm{Mev}$ or protons above $20 \mathrm{Mev}$. It was not possible, on the basis of the results of the single Geiger-Mueller tube, to determine which of the many possible atomic particles was responsible for the intense radiation field. Therefore, more definitive measurements were made in Explorer IV. The experiments were again under the direction of Dr. Van Allen, but the payload implementation (with the sole exception of the JPL Microlock beacon) was accomplished by the Army Ballistic Missile Agency. The scientific conclusions reported here are again due to $\mathrm{Dr}$. Van Allen. 
The cosmic-ray detectors in Explorer IV consisted of two small Geiger counters and two scintillation counters. One of the Geiger counters was unshielded and responded to any particle which could penetrate the $0.023-$ in. stainless steel shell of the satellite and the 0.05 -in. stainless steel casing of the Geiger tube. The other Geiger tube was shielded with 1/16-in. -thick $\left(1 \mathrm{~g} / \mathrm{cm}^{2}\right)$ lead sheet. One of the two scintillation counters recorded a pulse each time a particle passed through it; the other counter measured the energy of the particles which came to rest in it. Both of these scintillation counters were shielded by lead except for one window each, where the shielding was made as light as possible and placed next to a hole cut in the steel shell of the satellite.

The results of the first two Explorers were confirmed by the detectors in Explorer IV. Again the intense field of radiation at altitudes above $600 \mathrm{mi}$ was observed. The number of particles passing through the unshielded Geiger tube showed a density of about 12,000 particles $/ \mathrm{cm}^{2} / \mathrm{sec} / \mathrm{sterad.}$ At least $60 \%$ of these particles had enough energy to penetrate the lead shield around the other Geiger counter.

In addition to these very energetic particles, the scintillation counters revealed that there is an even greater density of particles with low energy. At the northern or southern limits of the trajectory these low-energy particles predominate. Here, the flux of particles of low energy at an altitude of about 1400 miles is about 80,000 particles $/ \mathrm{sec} / \mathrm{cm}^{2} / \mathrm{sterad}$.

To put this information in terms of another number, radiation intensities in excess of 100 roentgens/hr have been observed by the satellites, and this 
intensity increased steadily with altitude, with no indication of any leveling off at altitudes reached by the Explorers. It remained for the moon probes, Pioneers III and IV, to discover how far away from the earth the dangerous radiation belt extended and what the maximum counting rate might be.

\section{MICROMETEORITE INSTRUMENTATION AND MEASUREMENTS}

The measurements of micrometeorite densities in Explorers I and III were carried out under the direction of Dr. E. Manring and Dr: M. Dubin of the Air Force Cambridge Research Center. Two types of micrometeorite detectors were used in Explorer I: a parallel combination of twelve wire-grid detectors (Fig. 7) and an ultrasonic impact microphone, in spring contact with the steel shell, and its associated amplifier (Fig. 8).

In the grid-type detector, the severing of a wire in one of the grid structures by a micrometeorite of sufficient size and velocity resulted in a small step increase in resistance. This change of resistance, when sensed by the resistance-controlled oscillator, produced a step increase in subcarrier frequency. To assure accuracy, a flight erosion gauge with a multipleswitching network was used for calibration.

Frequency-vs-temperature data for the impact microphone was obtained by subjecting both the beacon and the microwhone equipment to oven and refrigerator temperatures. Thus, if the scaler output voltage varied with temperature, it was automatically included in the frequency calibration.

Ultrasonic microphone sensitivity was determined by dropping glass beads of known size and density on the satellite shell from various heights. 
With the height necessary to actuate the scaler and the size of the glass beads known, the required momentum averaged over the entire shell was found to be $0.012 \mathrm{~g}-\mathrm{cm} / \mathrm{sec}$. Therefore, if a velocity of $40 \mathrm{~km} / \mathrm{sec}$ (velocity of the earth in space) is assumed for a micrometeorite, the minimum detectable particle mass is found to be $3 \times 10^{-9} \mathrm{~g}$. As an additional check, shaped explosive charges were fired at the steel shell to simulate high-velocity-particle impact.

In the data which have been reduced from the Explorer I high-power transmitter, which carried data from the uitrasonic microphone, 38 impacts were observed from January 31 to February 12, 1958. This result indicates that the average influx of particles 4 microns or more in diameter (one-fifth the diameter of human hair) was approximately 0.01 particle $/ \mathrm{cm}^{2} / \mathrm{sec}$. Almost all impacts were observed between midnight and noon, satellite local time. Only on one day out of twelve were hits observed at any other time, and then between 2 and 7 p.m. These results show that the average speed of the dust particles in space is less than the speed of the earth in its orbit, so that the earth appears to be sweeping through a cloud of dust. Only on one day was a shower recorded in which the speed of the particles was greater than that of the earth, allowing them to catch up to the earth from behind. Daily variations in flux up to a factor of 10 were observed.

The low-power transmitters on both Explorers I and III carried the data from the wire gauges, consisting of a set of twelve cards (each $1 \mathrm{~cm}^{2}$ in area) wound with two layers of fine wire. No more than one (and perhaps none) of the wire gauges was broken during the lifetime of the telemetering system on 
Explorer I. This information permits an upper limit to be established for the influx of particles of 10 microns diameter or larger. This limit is 0.001 particle $/ \mathrm{m}^{2} / \mathrm{sec}$ during the lifetime of the experiment, from January 31 to April 14.

Data received from Explorer III showed no wire gauges broken from March 26 until May 6. Between 2243 Greenwich Mean Time on May 6 and 0232 GMT on May 7, two of the wire gauges were fractured. Then both transmitters of Explorer III started to function erratically about May 8, only about 6 weeks after launching and long before expected battery exhaustion. The signals became erratic at the same time on both transmitters, which was very curious, since the two transmitters were completely independent. These symptoms were consistent with an assumption of physical damage. All of the micrometeorite data before this time was taken in a period of normal background activity. No meteor showers were encountered. However, the shower Eta Aquarides, which has been associated with Halley's Comet, occurs during the early part of May and reaches its most intense activity on about May 6 .

\section{TELEMETERING SUBCARRIER OSCILLATORS}

Both the resistance-controlled and current-controlled subcarrier oscillators used in the Explorer satellites were self-contained (Fig. 9), using four mercury cell batteries each. Each consumed about $2.5 \mathrm{mw}$. The type of resistance-controlled oscillator used in Explorer I is shown in Fig. 9a. The current-controlled oscillator, shown in Fig. 9b, used time-controlled 
reactance modulation for controlling oscillator frequency. This method was made possible by the characteristics of the transistor. The operating frequency of a conventional L-C oscillator can be altered by introducing an alternating current that is of the same frequency but is 90 deg out of phase with the oscillator voltage. The frequency shift thus produced is proportional to the amount of additional current introduced into the tuned circuit.

Oscillator frequency shift can be obtained using a transistor as a timecontrolled reactance modulator. The portion of the cycle during which this reactive current is introduced can be controlled by direct current introduced into the control transistor. By this new method, frequency modulation is achieved with minimum dependence upon temperature-sensitive parameters.

\section{MICROLOCK COMMUNICATIONS SYSTEM}

Beginning in 1953, the Jet Propulsion Laboratory studied and developed simple light-weight radio transmitters and ultra-sensitive receivers. Weighing only about $1 \mathrm{lb}$ with their batteries, the tiny transmitters could serve in small . satellites as continuous tracking beacons for about 2 months. It was even possible to add several FM telemetry channels to the carrier signal (Fig. 5) to convey environmental data. It also was necessary to use highly sensitive and complex receiving equipment on the ground. A phase-locked receiver developed by JPL for Army missile instrumentation purposes was adapted for the purpose. This combination of a minimum-weight transmitter with a phase-locked ground receiver was named the Microlock communications system (Fig. 10). To permit 
tracking of satellites approaching from any direction, a helical quasiomnidirectional receiving antenna was used.

Microlock tracking stations, located on the east and west coasts of the Americas, Nigeria, and Singapore, picked up the signals and recorded them together with accurate time signals on magnetic tape. These tapes were then flown to various data reduction centers, the principal one being at the Jet Propulsion Laboratory in Pasadena. When the magnetic tapes were received at these centers, the telemetered data was reduced onto Sanborn tapes, advancing at a $1-\mathrm{mm} / \mathrm{sec}$ rate. An analog record (Fig. 11), as well as digital printouts of counted audio frequencies, was thus made available. The reduced data were then turned over to IGY scientists familiar with the characteristics of the instruments on board the satellite. They, in turn, could interpret the frequency measurements in terms of the actual measured quantities which had been observed by the satellite in its orbit above the earth.

\section{MICROLOCK GROUND RECEIVER}

The performance of any signal-detection system can be maximized only by using all available a priori information concerning signal frequency, strength, polarization, and bandwidth, as well as receiver noise level. One means of employing some of the a priori information about the most probable received signal characteristics from a rapidly moving satellite is correlation detection. The method of mechanizing correlation detection used by JPL in the Microlock receiving system is the phase-locked loop (Fig. 12), which is particularly applicable when the transmitted signal is a pure sine wave. In the phase-locked 
loop the local estimate of the transmitted signal is generated by a voltagecontrolled oscillator ( $\mathrm{VCO}$ ), and the mathematical operation of multiplication is performed by the phase detector. This phase detector provides a dc output voltage which is proportional to the phase difference between the incoming signal and the local estimate generated by the VCO. The dc error voltage is separated from noise by the low-pass filter (integrator) to provide a control voltage to the VCO. In this way the phase-locked loop is an electronic servomechanism which accomplishes signal detection using only linear mixing. This system provides memory by virtue of the voltage stored on the integrating capacitor in the lowpass filter. This voltage is always a "best guess" of the present signal frequency and is retained for some seconds after disappearance of the signal, thus providing a means for re-acquiring the signal should it appear again at the same frequency. The design of a phase-locked loop for a particular application requires detailed knowledge of target dynamics and their effect on signal characteristics, since the dynamic tracking characteristics and noise bandwidth of the phase-locked servo loop are affected.

In a second channel, the signal is shifted in phase by $90 \mathrm{deg}$, so that it is in phase with the VCO output. The phase detector in this channel then produces a dc voltage which is proportional to the signal amplitude. In this manner, the signal is completely detected by the phase-locked loop; i. e., the frequency, phase, and amplitude are determined.

In this description of the phase-locked loop, it is assumed that the loop is initially locked. Initial acquisition of the signal may be accomplished by 
slowly sweeping the VCO frequency across the signal frequency. As the beat between the signal and the VCO output goes to zero, the system acquires phase lock and thereafter retains synchronism unless the signal level becomes so small that the available control voltage can no longer overcome the effects of the small amount of noise which appears at the output of the low-pass filter.

\section{SATELLITE TRANSMITTERS}

Two similar but independent transmitters (Fig. 13) were used in each of the Explorers. This arrangement provided additional reliability in the form of redundancy so that orbit parameters could be accurately determined. One transmitter operated at $108.03 \mathrm{mc}$ with an output of $60 \mathrm{mw}$, and the lower power unit operated at $108.00 \mathrm{mc}$ and $10 \mathrm{mw}$. Information on the space environment was sent back on four standard FM/FM telemetry channels. The frequency of the audio signal was modulated by changes of resistance or current in the measuring instruments in the satellite.

The higher power (60 $\mathrm{mw}$ ) unit, using amplitude modulation, made it possible for nonprofessional radio receiving equipment around the world to record telemetry signals of cosmic events as they occurred while the Explorers were above the local horizon. Because of the higher power drain, the mercury battery power supply could operate this transmitter continuously for only about 2 weeks, but the 10-mw Microlock transmitter could provide telemetry and tracking data for over 2 months. This tiny 10-mw unit was continuously heard by Microlock tracking stations for as long as 35 min during a pass near the satellite apogee altitude of $1500 \mathrm{mi}$. 
Figure 13a shows a schematic diagram of the low-power Microlock transmitter. This 10-mw transmitter is powered by a battery of six mercury cells from which it draws approximately $5 \mathrm{ma}$ current at about 8 volts. Phase modulation of the carrier signal with four standard FM/FM channels of telemetry is produced by varying the voltage applied to the collector of oscillator $Q_{1}$. This circuit produces in the stable crystal-controlled oscillator about a 14-deg index of phase modulation. A conversion efficiency of $25 \%$ was obtained with class $\mathrm{C}$ operation. The high-power transmitter of Fig. $13 \mathrm{~b}$ is similar to the low-power unit. Amplitude modulation is produced by varying the voltage applied to the collector of the doubler-buffer transistor. An audio voltage of about 8 -volt peak produces a $50 \%$ amplitude modulation of the carrier signal.

Some Explorer transmitters stopped operation at the expected time of battery exhaustion, only to return to the air again after a few days of silence. This phenomenon was due to the use of duplicate battery supplies. When the larger battery for the transmitter was power-depleted but still capable of producing a back voltage, oscillations stopped. However, dc current continued to flow until complete exhaustion of the battery, and thus a true short circuit was produced. Thereupon the other battery, which still had some residual power, started transmitter operation on a different mode.

\section{SATELLITE ANTENNAS}

In Explorer I, the output of the high-power transmitter was lead through a phasing and matching network into four flexible-wire turnstile antennas, mounted perpendicular to the cylindrical case at 90-deg intervals. The nearly 
omnidirectional radiation pattern thus produced was believed desirable because of the random payload orientations. Some pattern dissymmetry made it possible to determine satellite spin rate from received RF signal strength records.

During the second orbital pass of Explorer I, a very slow periodic fluctuation in signal strength became noticeable, in addition to the flutter caused by the 750-rpm rotation rate. By the end of the first orbit it was already evident that the 750-rpm axial rotation was being damped out. A slow-precession soon transferred the rotation axis to an end-over-end tumble with a 9 -rpm rate. The change in motion was due to mechanical energy losses in the whip antennas under the condition that satellite momentum had to be conserved. To reduce the large signal-strength fluctuations resulting from this changing motion so early in the broadcasting lifetime, it was decided to eliminate whip antennas in Explorer III. Instead, the rocket motor case was electrically matched to the instrument carrier case to act as a dipole radiator. Explorer III did not begin to tumble until the end of the third day, after its orbital parameters had been firmly established.

The tumbling phenomenon had a valuable by-product. In explaining the signal-strength variations, an analytical technique was developed which permits a surprisingly accurate determination of vehicle attitude using only ground measurements. The technique has been exploited in describing the performance of the high-speed rocket cluster and in specifying final spin orientation of the Explorer and Pioneer payloads. The tumbling action also served to demonstrate the remarkable property of the Microlock system to perform well even under 
comparatively severe signal-strength fluctuations, a design criterion derived from the previous JPL telemetering philosophy.

Both scientifically and technologically, the Explorer electronics was considered successful.

\section{PREFLIGHT TESTING}

No statistical information concerning the expected reliability of the new component units was available at the time of the Explorer flights. The severe environmental specifications and the high reliability required for the Explorer satellite instrumentation prescribed that all components be subjected to $100 \%$ flight-acceptance and type-approval tests. Flight-acceptance tests, applied to all units prepared for flight, including spares, were designed to subject the components to an environment simulating that encountered in space flight. The type-approval tests, applied to a sample payload, were designed to verify that the payload design was capable of surviving an environment more severe than that expected, as well as surviving the flight-acceptance test and then the space flight.

The second phase of the testing program consisted of subjecting the payload shell to temperatures fluctuating between $-30^{\circ}$ and $120^{\circ} \mathrm{C}$, an environment similar to that expected during flight. A safe temperature cycle of several hours demonstrated that the internal temperature variation was within the range desired, 0 to $45^{\circ} \mathrm{C}$. Noise vibration of $15 \mathrm{~g}$ simulated the vibration produced by the burning of the fourth-stage rocket motor. Centrifuge tests to $100 \mathrm{~g}$ were used 
Technical Release No. 34-12

to simulate the linear acceleration during the firing of the fourth-stage motor. Spin tests at $750 \mathrm{rpm}$ duplicated the expected final-stage spin rate. Simulated altitude tests in the vacuum chamber were used to check out the high-voltage circuitry. In this test it was found necessary to provide additional high-voltage breakdown protection in the form of plastic foam potting. 


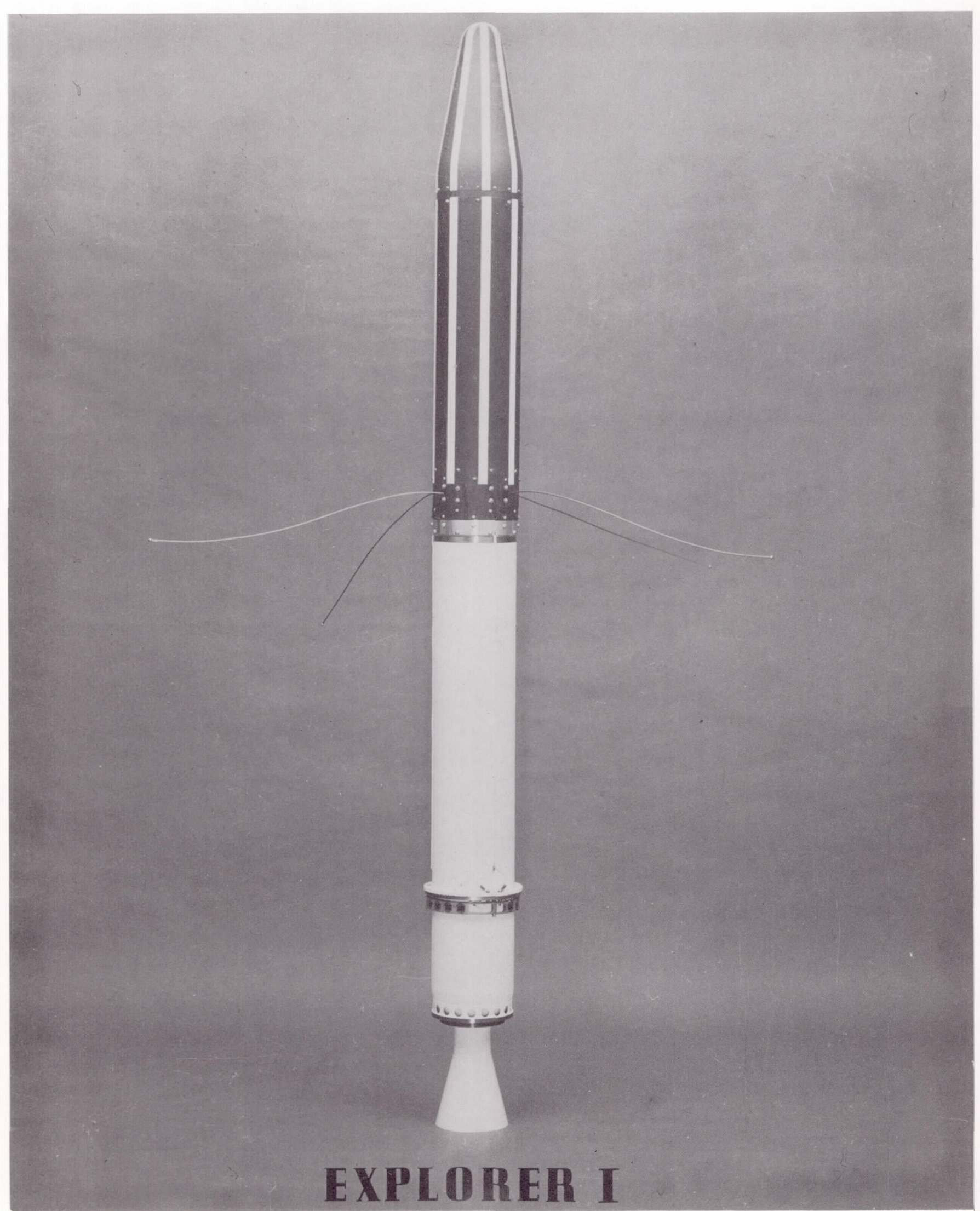

Fig. 1. The United States' First Earth Satellite, Explorer I 
Technical Release No. 34-12

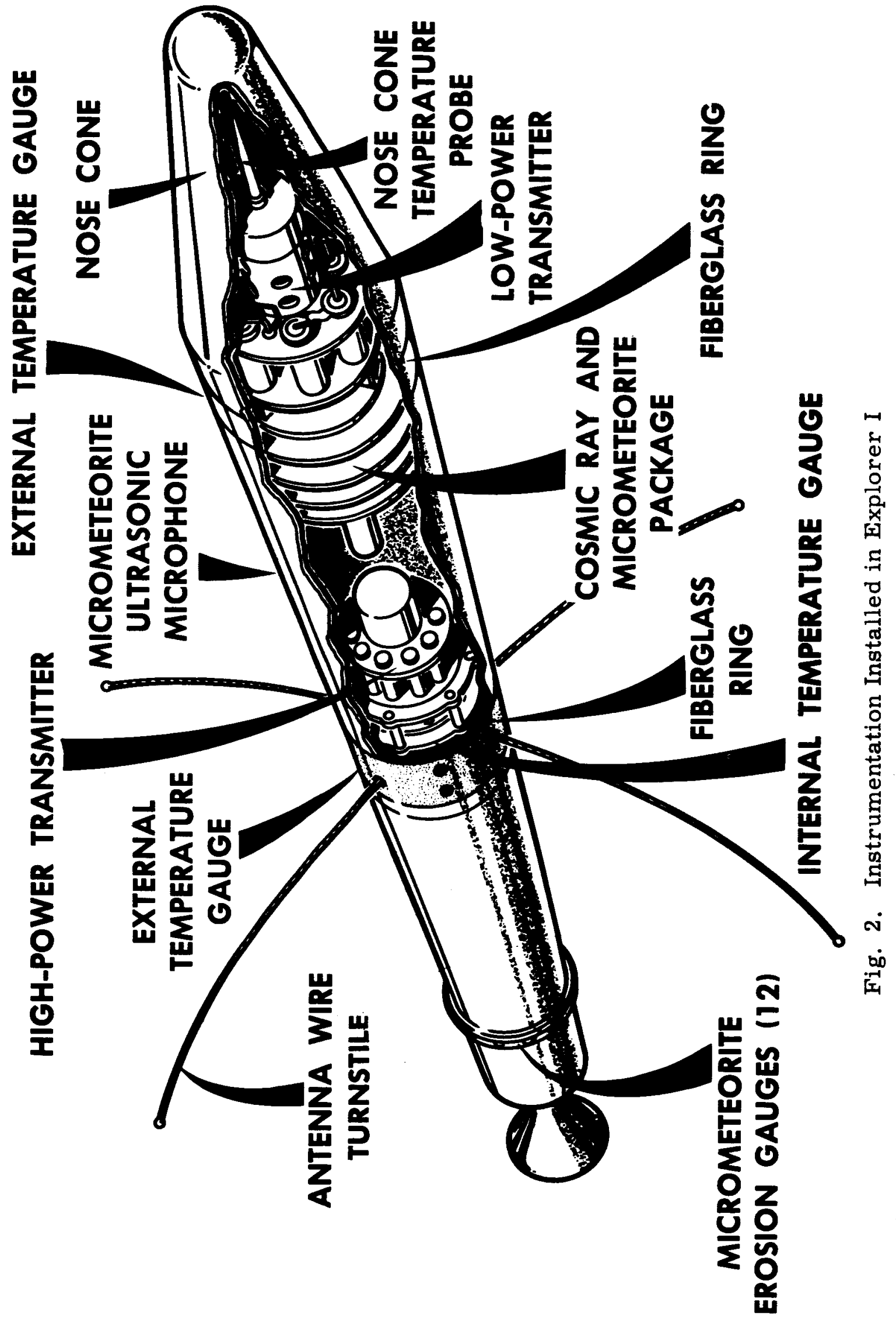

Page 21 
Technical Release No. 34-12

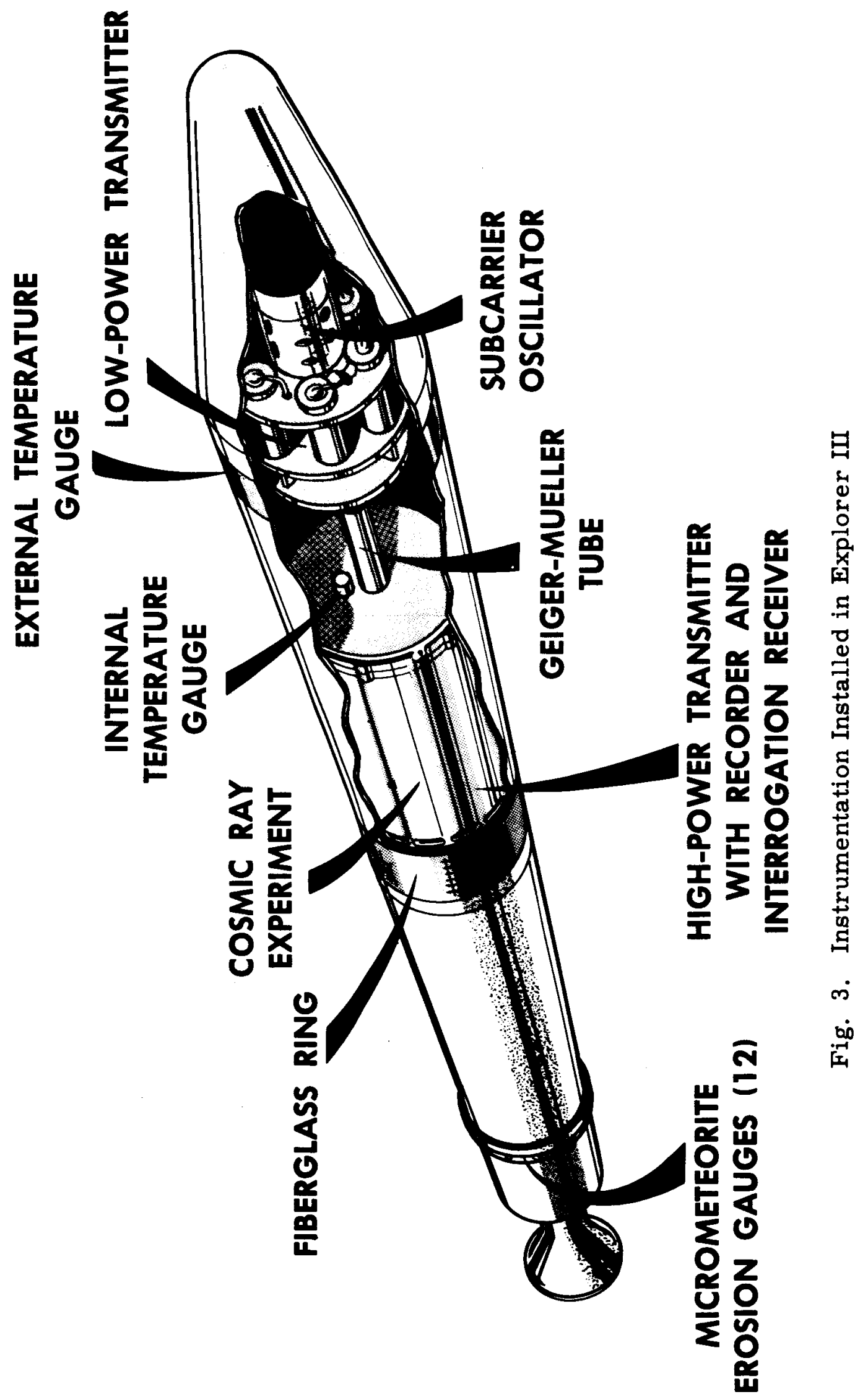




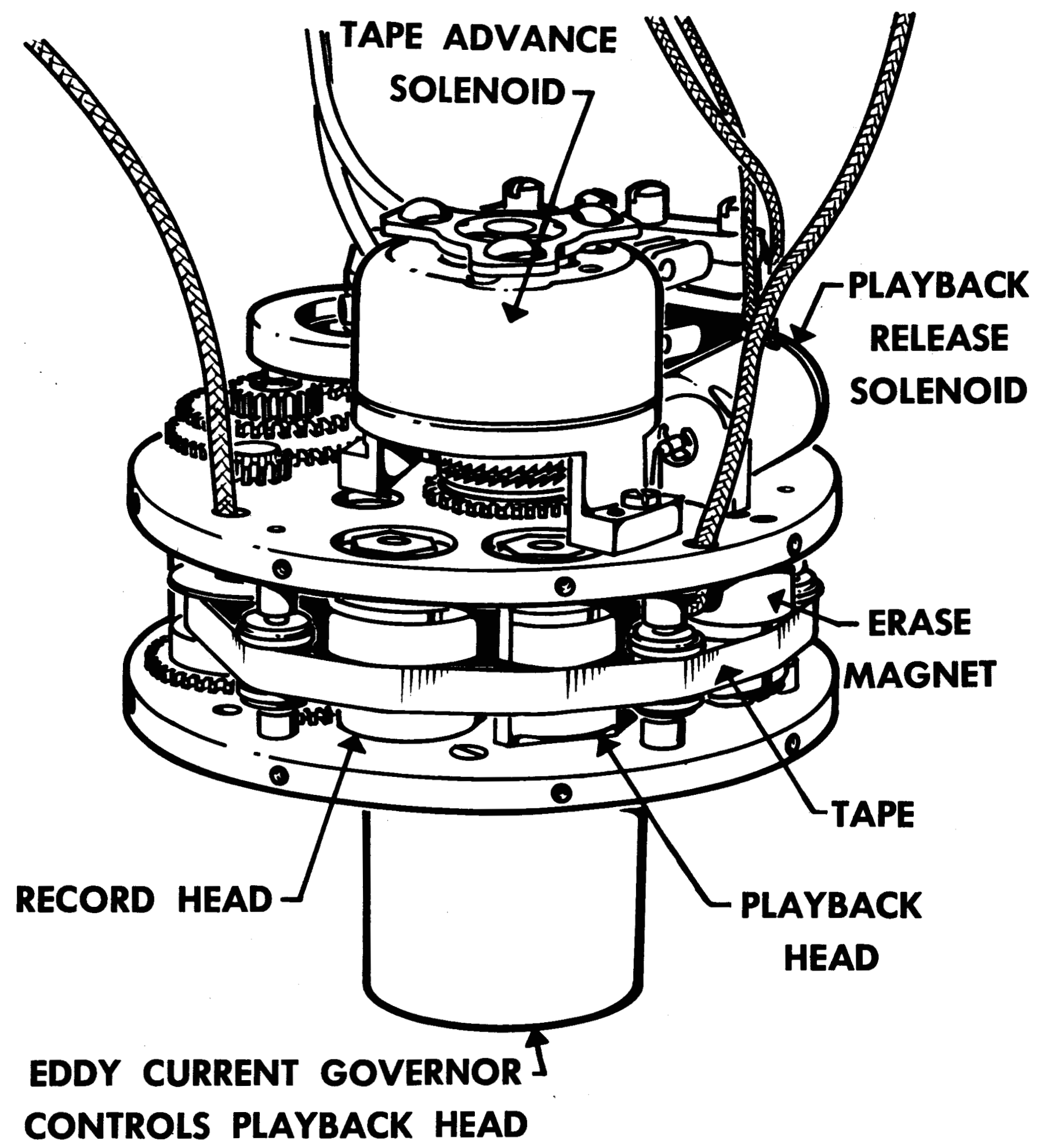

Fig. 4. Tape Recorder for Cosmic-Ray Data Flown in Explorer III 

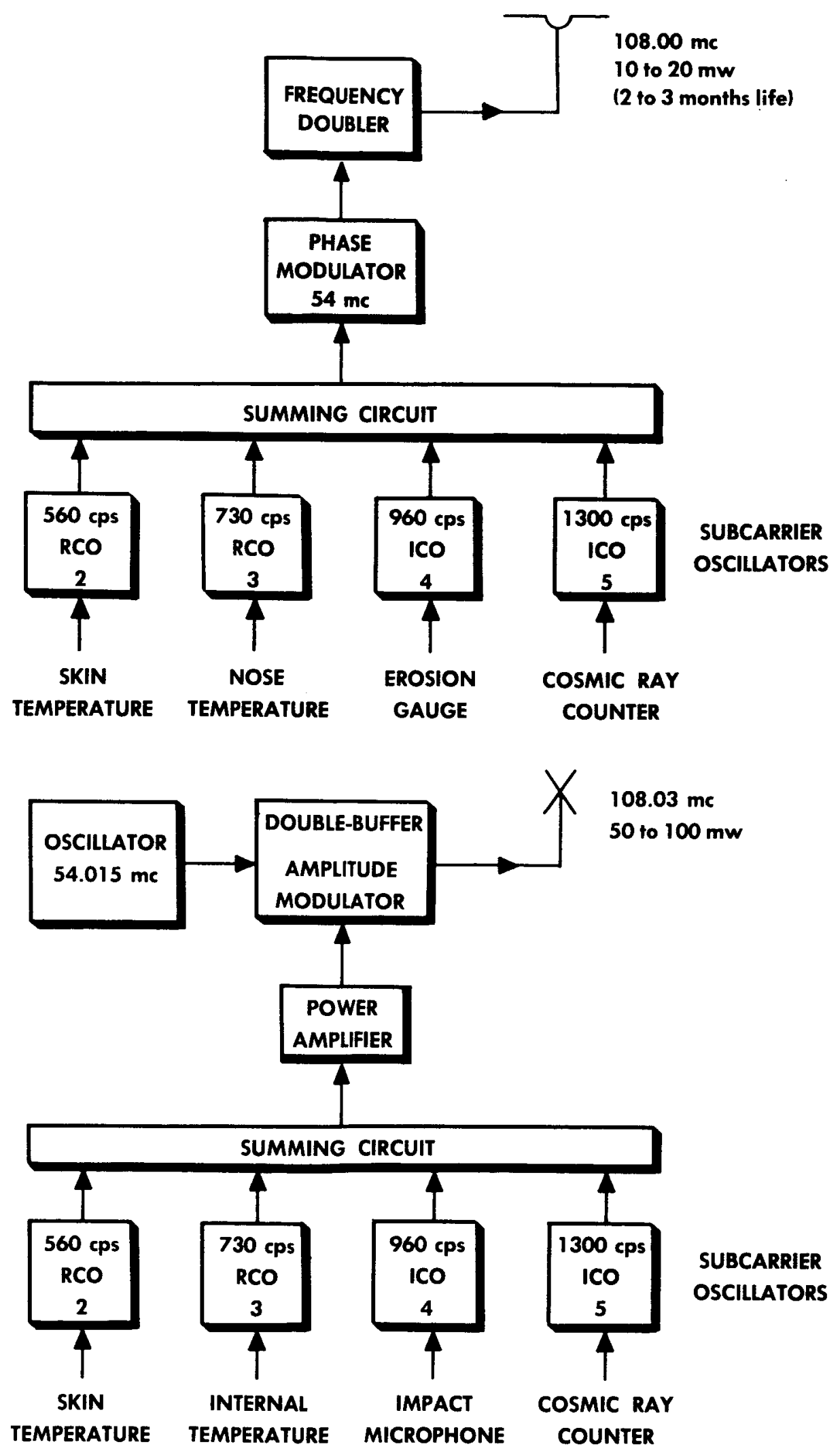

Fig. 5. Telemetering Systems Flown in Explorer I 


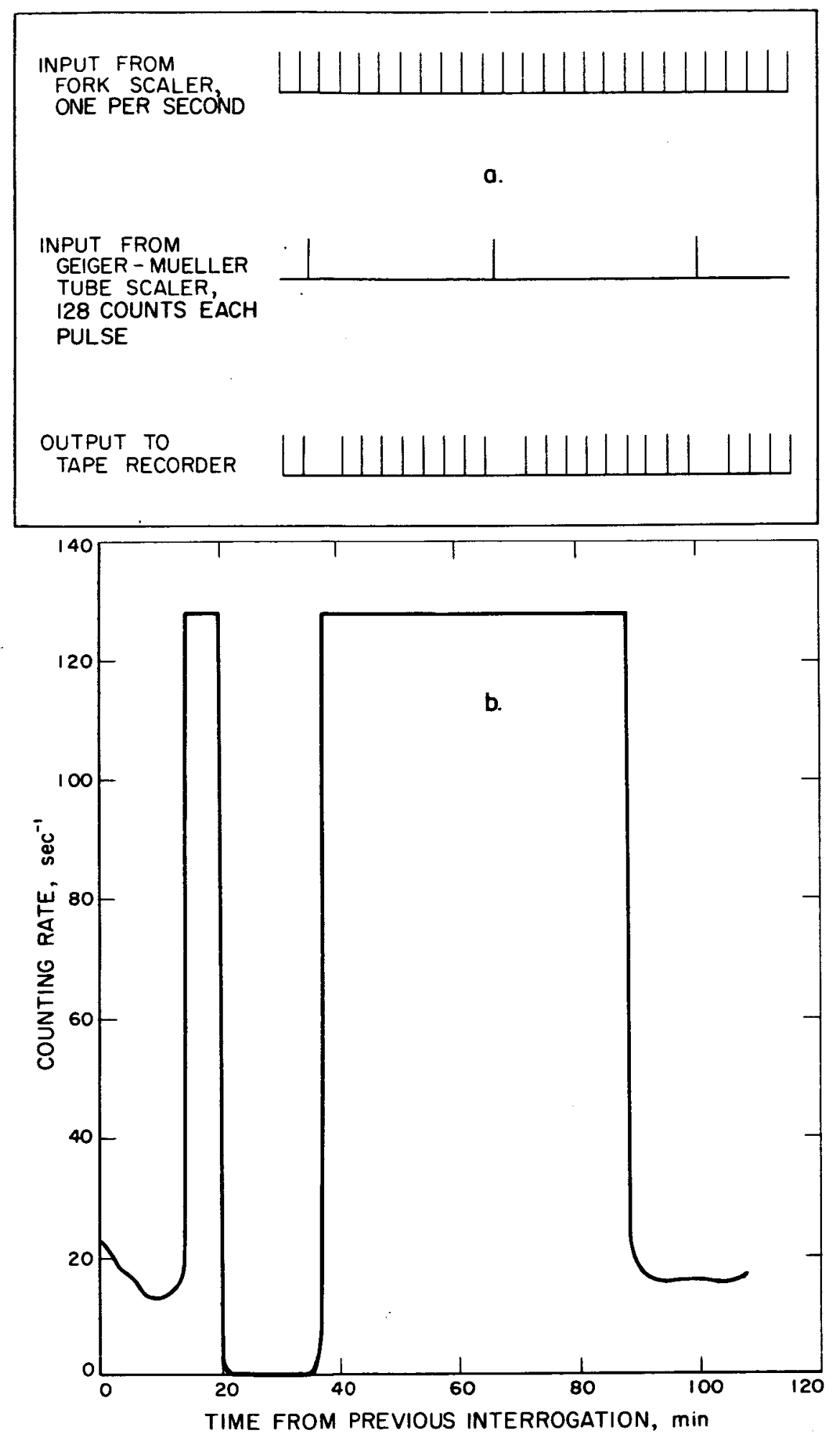

Fig. 6. Cosmic-Ray Data: (a) Pulse Inputs to Recorder; (b) Typical Reduced Counting Rates for One Complete Orbit 


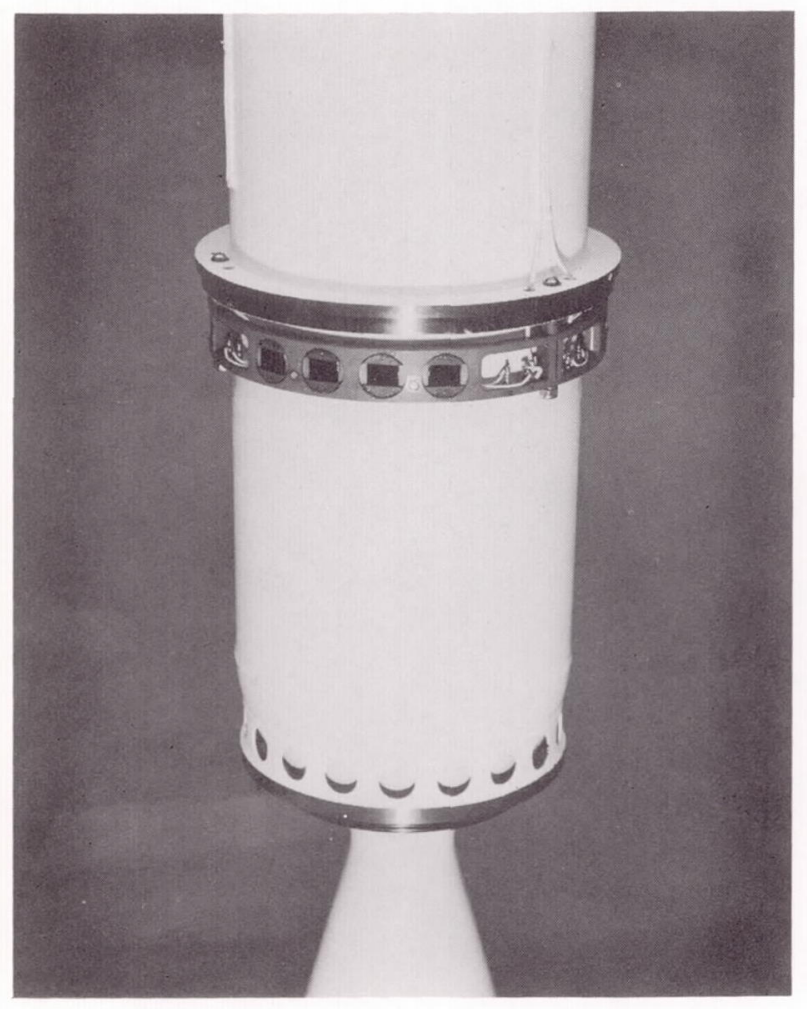

Fig. 7. Wire-Gauge Micrometeorite Detector

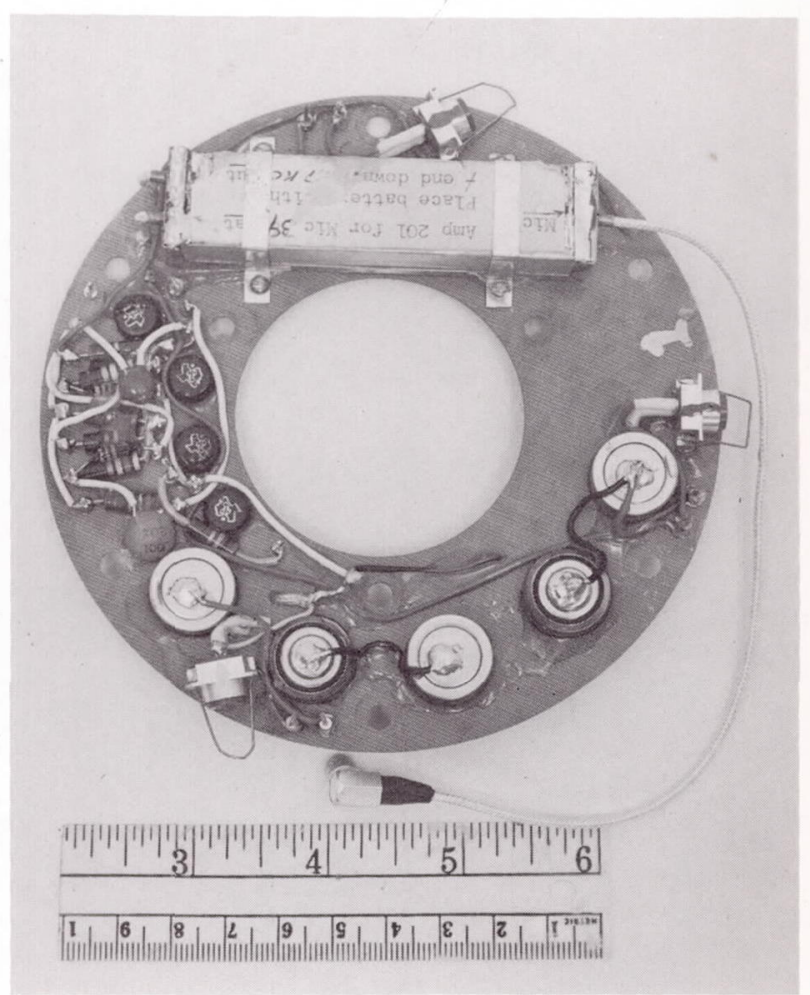

Fig. 8. Micrometeorite Microphone and Amplifier 

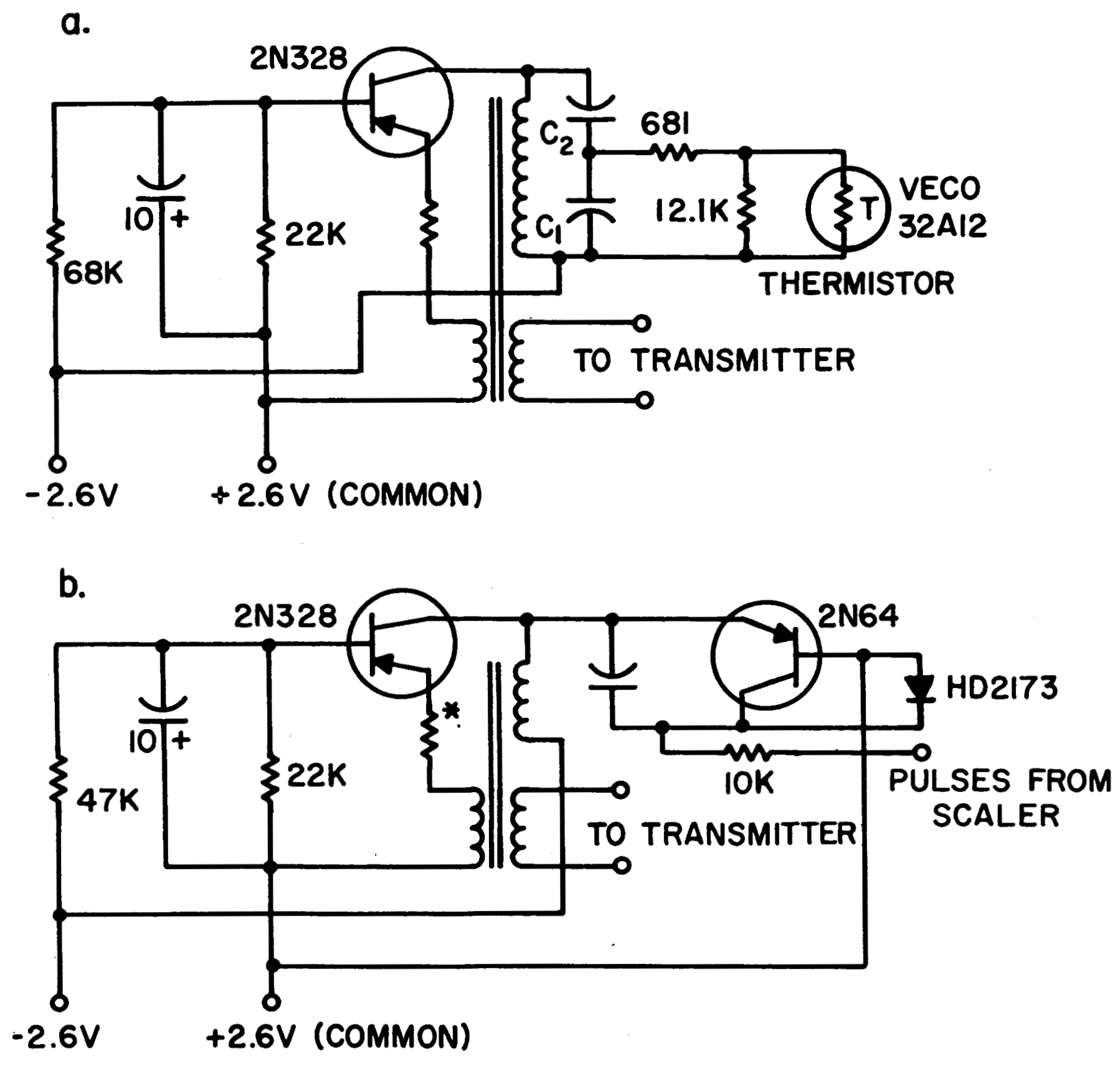

* value $20 \%$ below that for oscillation

Fig. 9. Subcarrier Oscillators Used in Explorer Satellites 
Technical Release No. 34-12
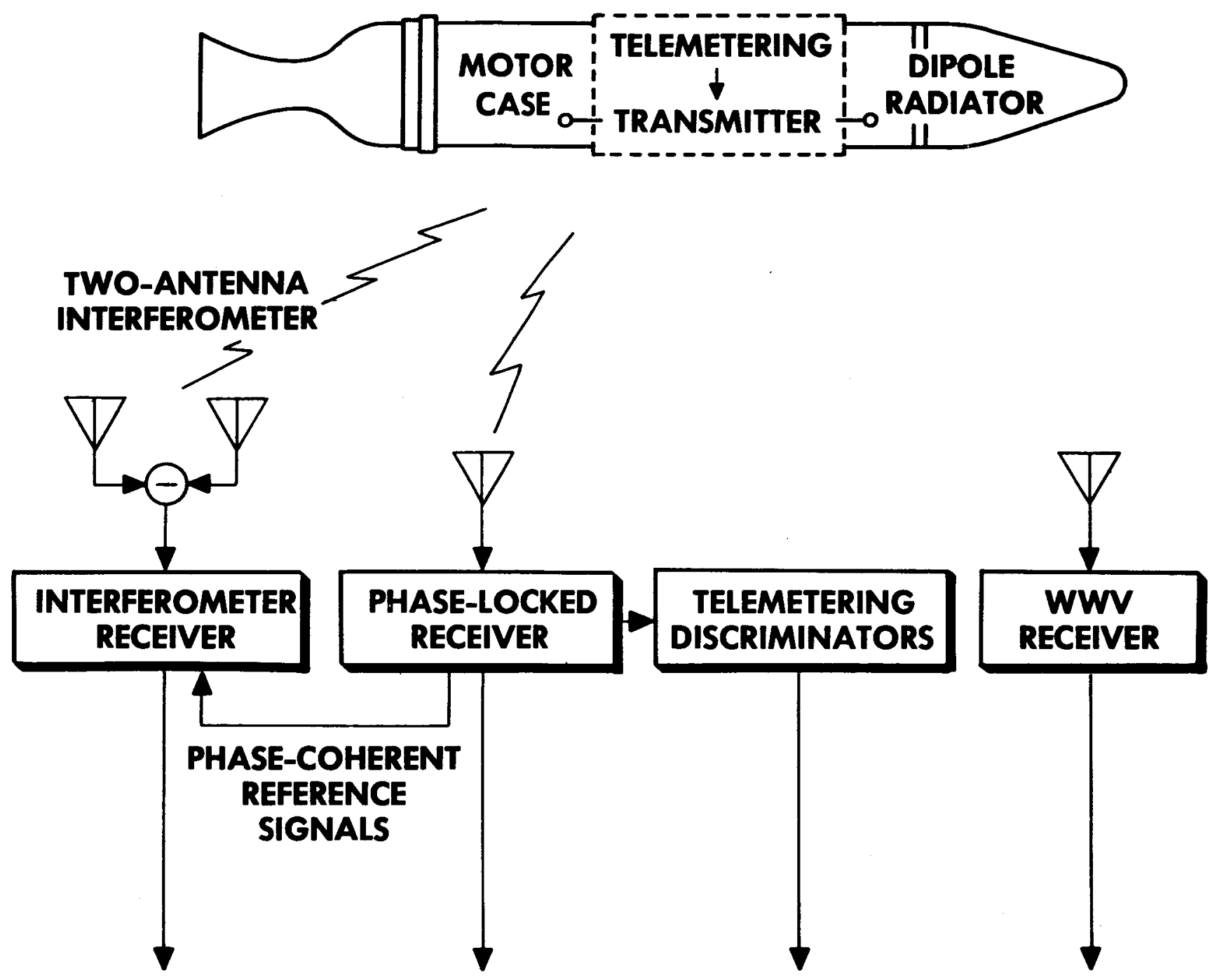

ANGLE OF ARRIVAL

FREQUENCY AND SIGNAL STRENGTH
TELEMETERED

DATA
FREQUENCY AND TIME STANDARDS

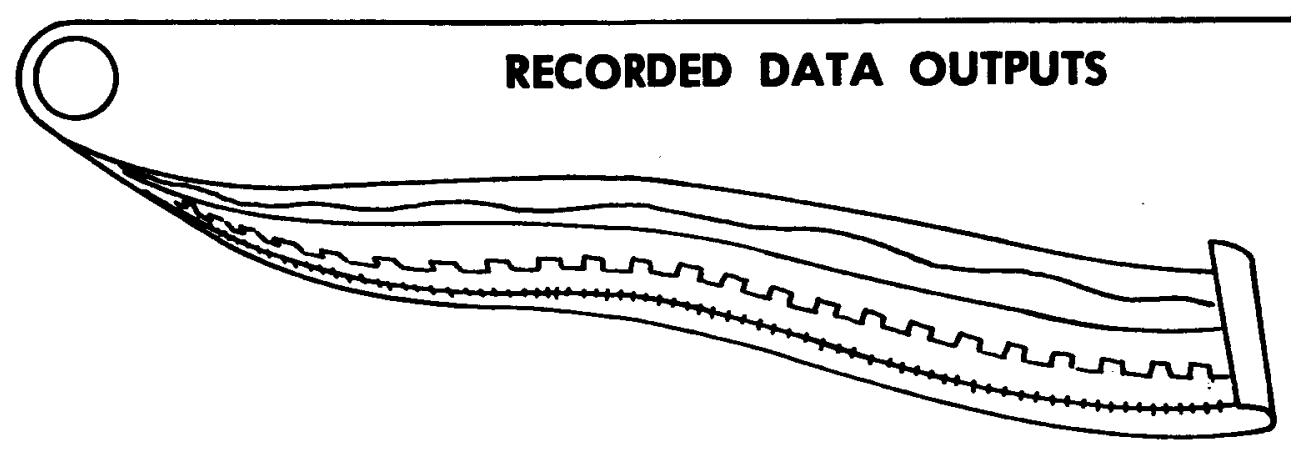

Fig. 10. Microlock Communications System Used in Tracking Explorer Satellites 


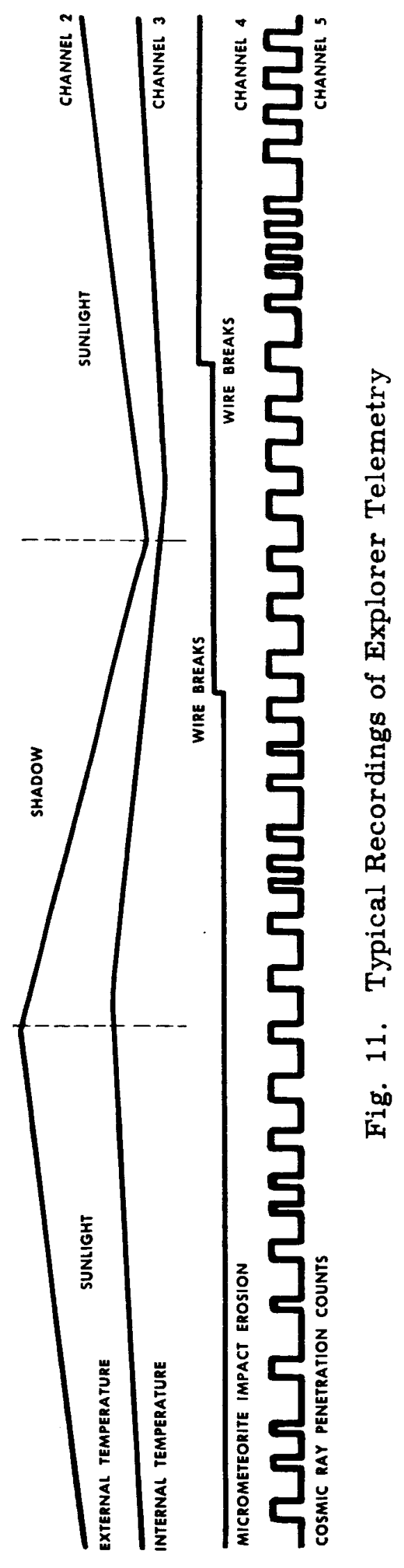




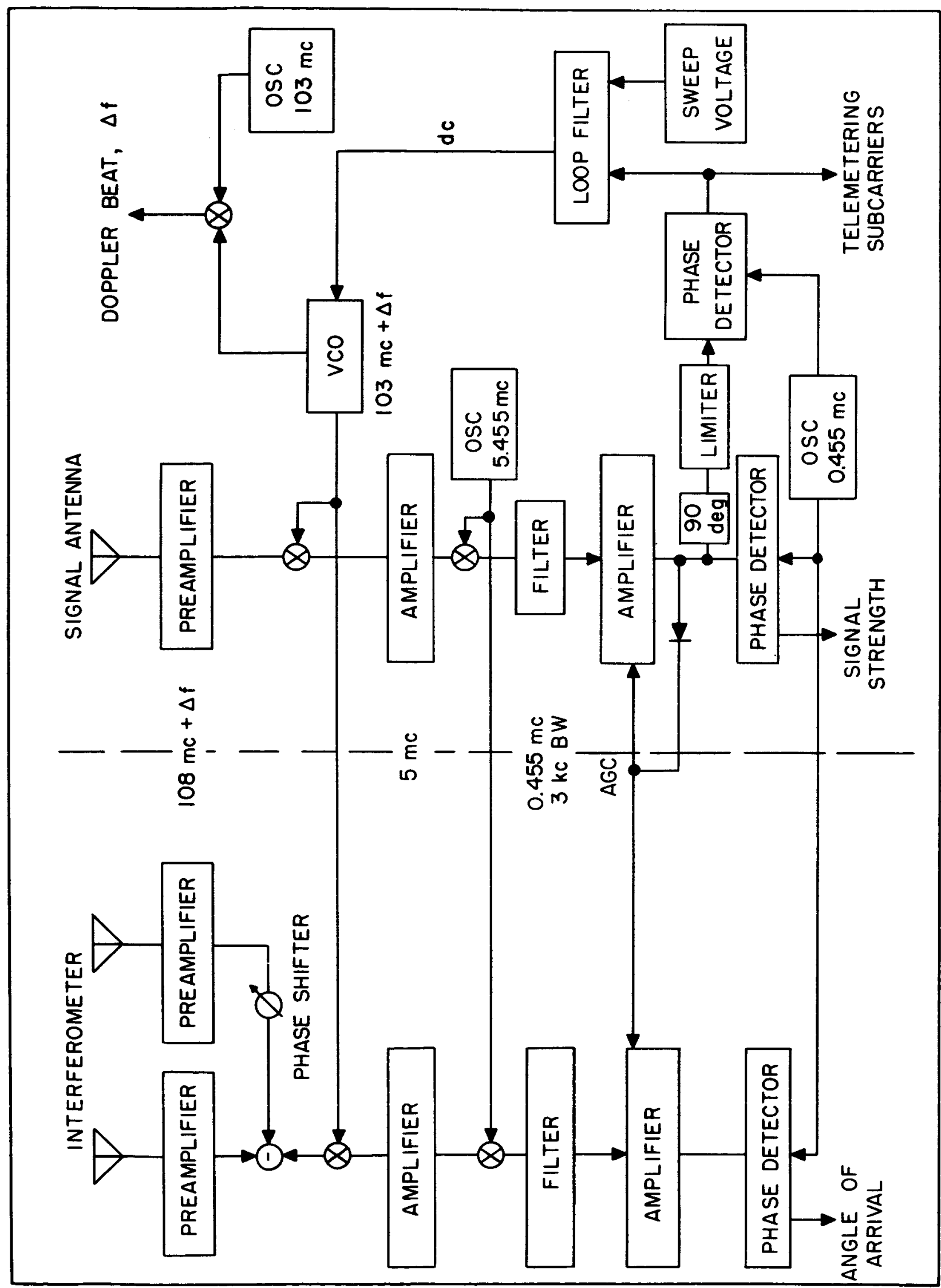

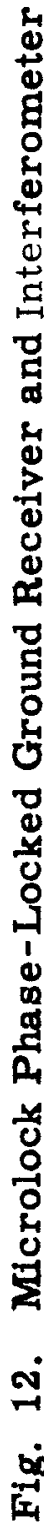


Technical Release No. 34-12

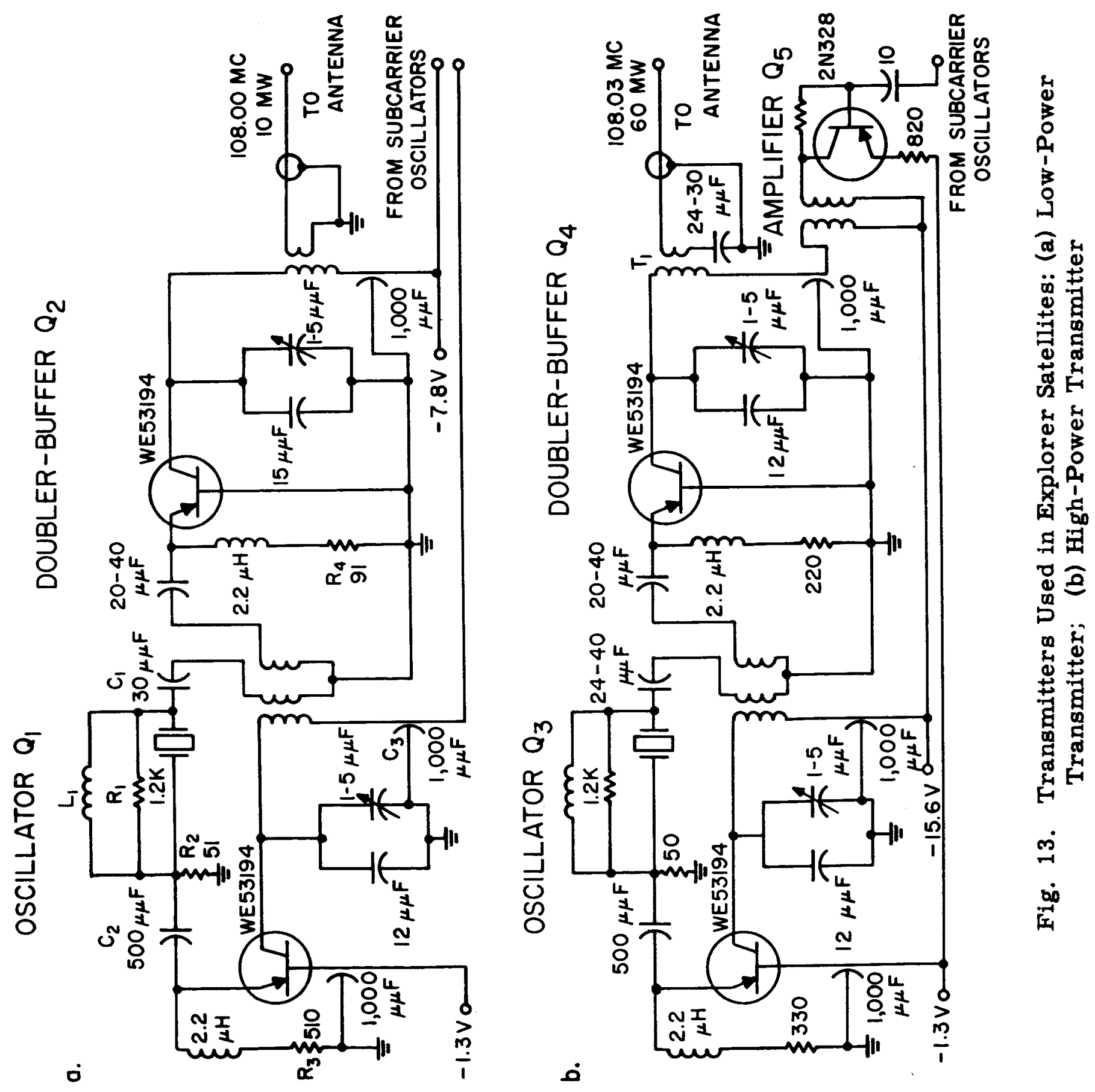

